\title{
An Atypical Presentation of Extragenital Lichen Sclerosus et Atrophicus
}

\author{
Sabina Vaccari ${ }^{1}$, Alessia Barisani ${ }^{1}$, Francesca Pepe ${ }^{1}$, Carlotta Baraldi ${ }^{1}$, \\ Bianca Maria Piraccini ${ }^{1}$, Valeria Gaspari ${ }^{1}$
}

1 Dermatology, IRCCS Policlinico di Sant'Orsola, Department of Experimental, Diagnostic and Specialty Medicine, Alma Mater Studiorum Università di Bologna, Italy

Key words: extragenital lichen sclerosus et atrophicus, morphea, dermoscopy, histopathology

Citation: Vaccari S, Barisani A, Pepe F, Baraldi C, Piraccini BM, Gaspari V. An atypical presentation of extragenital Lichen Sclerosus et Atrophicus. Dermatol Pract Concept. 2021;11(4):e2021072. DOI: https://doi.org/10.5826/dpc.1104a72

Accepted: January 29, 2021; Published: October, 2021

Copyright: (02021 Vaccari et al. This is an open-access article distributed under the terms of the Creative Commons Attribution License BY-NC-4.0, which permits unrestricted noncommercial use, distribution, and reproduction in any medium, provided the original authors and source are credited.

Funding: None.

Competing interests: None.

Authorship: All authors have contributed significantly to this publication.

Corresponding author: Francesca Pepe, MD, Dermatology, Department of Experimental, Diagnostic and Specialty Medicine, University of Bologna. Email: francesca1pepe@gmail.com

\section{Introduction}

Lichen Sclerosus et Atrophicus (LSA) is a chronic inflammatory, invalidating disorder mainly involving the anogenital skin. Extragenital lesions are uncommon, occurring as multiple, oval, porcelain-white macules, or papules. When present, they are mostly observed on the superior trunk, axillae, buttocks, and extremities, following Blaschko lines [1]. In the literature, there is a high prevalence of coexistence of LSA and morphea [2].

\section{Case Presentation}

We report the case of a 70-year-old woman who presented bilateral symmetrical itchy lesions affecting the inner thighs, appearing as wrinkled, porcelain-white color plaques with cigarette-paper-like texture and measuring $4 \mathrm{~cm} \times 4 \mathrm{~cm}$ (Figure 1A). The lesions had been present for about 1 year. Moreover, an involvement of the vulvar mucosa, with loss of clitoris and fusion between the labia majora and minora, was observed, as it happens in genital lichen sclerosus et atrophicus (LSA) (Figure 2A).

Dermoscopy of cutaneous lesions revealed widespread whitish patches, comedo-like openings, and peppering patterns (Figure 1B). On dermoscopy, the vulvar lesions showed patchy structureless milky-pinkish areas with scales and red purpuric blotches.

The differential diagnosis of the skin patches included morphea and extragenital LSA, due to their clinical morphology and distribution and to the association with anogenital lesions [3]. A punch biopsy of cutaneous lesions showed a thick hyperkeratotic scale and an atrophic epidermis with flattening of the rete ridges. The basal layer cells showed hydropic degeneration. Beneath the epidermis there was a broad area of pronounced lymphedema. Within this zone, the collagenous fibers were swollen and homogenous and contained only a few nuclei. Dermal melanophages, rare lymphocytes, and plasma cells were observed (Figure 1C). Based 


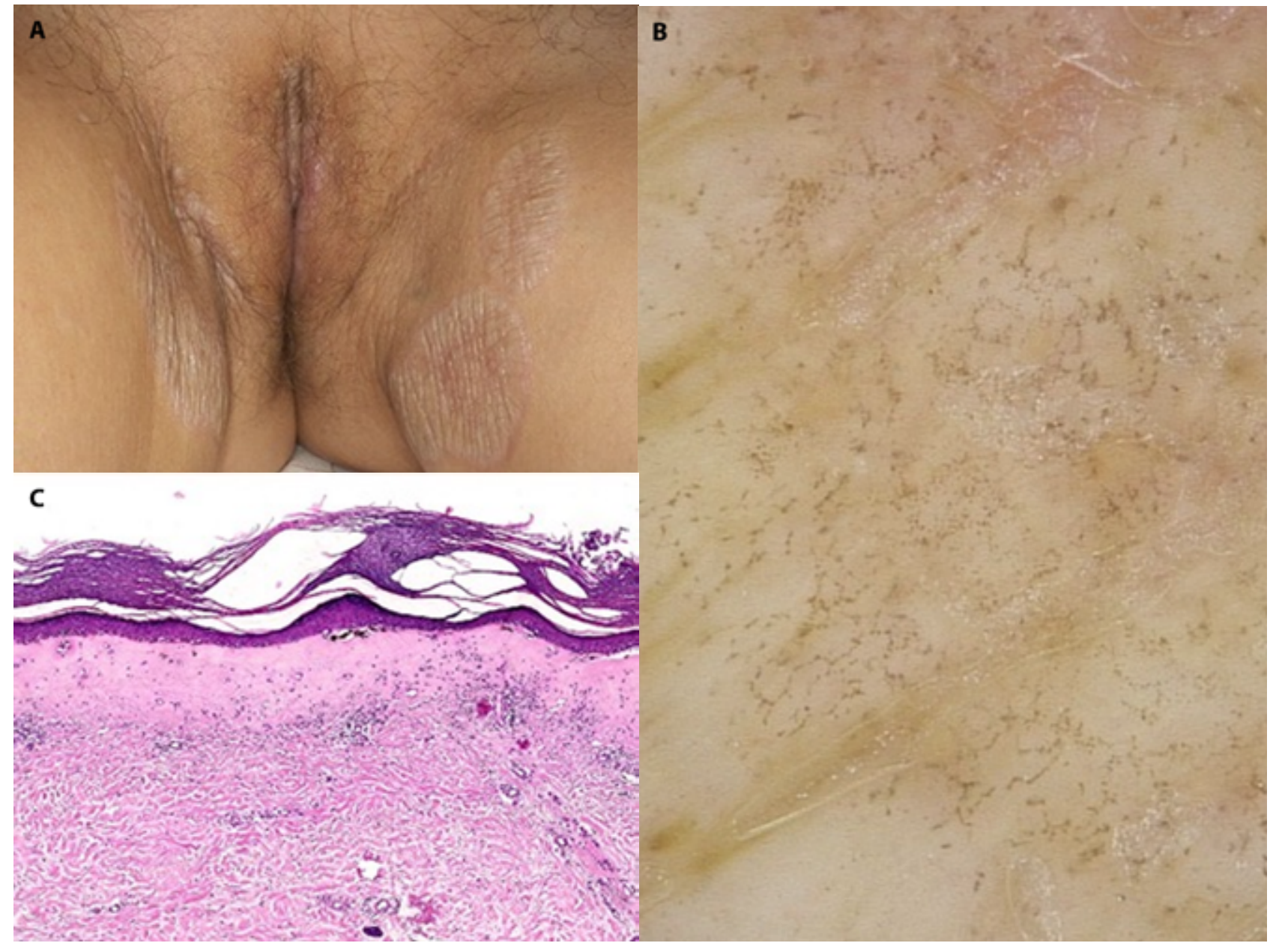

Figure 1.(A) Extragenital lichen sclerosus et atrophicus with porcelain-white color plaques. (B) Dermoscopic patterns: abundant peppering over white-yellowish structureless areas (magnification x 20). (C) Histological examination: thick hyperkerator scale an atrophic epidermis and a pale superficial dermal stroma with rare lymphocytes and plasma cells; melanophages displaced in upper dermis.

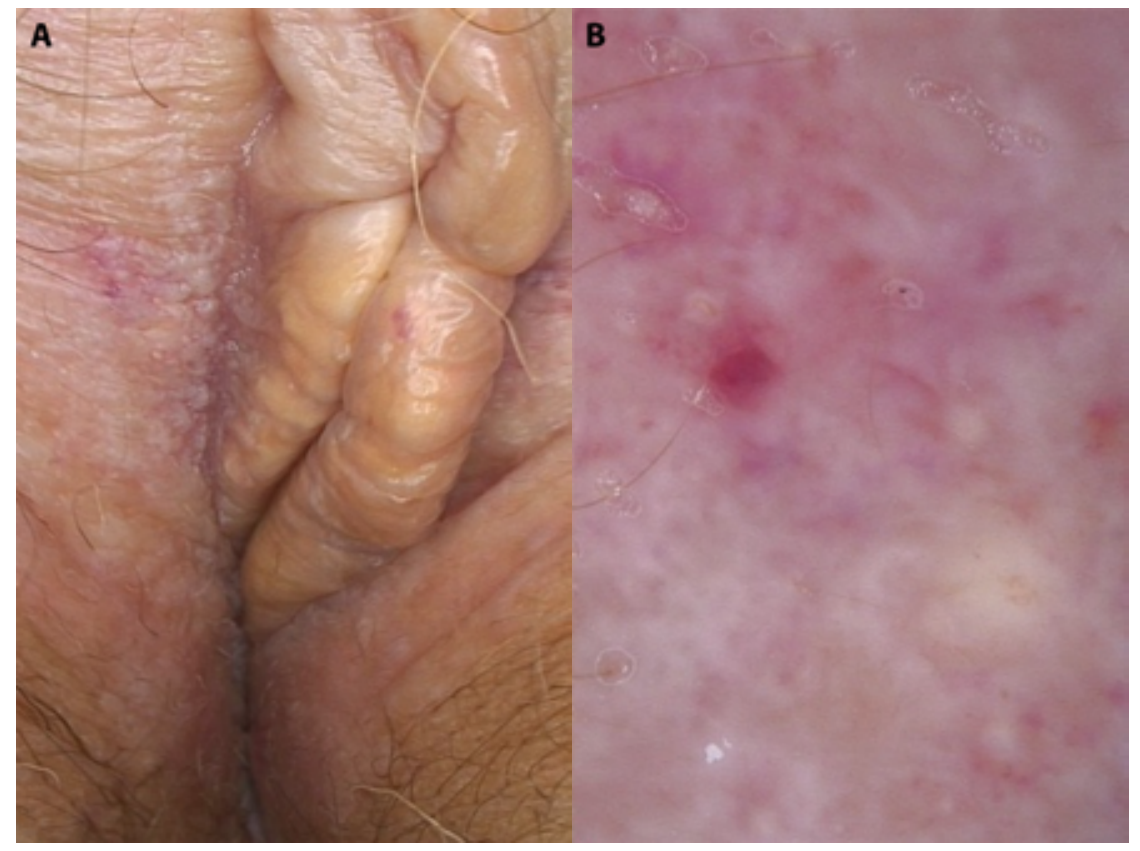

Figure 2. (A) Genital lichen sclerosus et atrophicus with patches of sclerosis and purpuric lesions. (B) Dermoscopic patterns: red purpuric blotches corresponding to ecchymosis in an intensely white background . 
on the clinical and histological findings, a final diagnosis of extragenital LSA was made. A vulvar biopsy was also performed, confirming the diagnosis of genital LSA (Figure 2B).

\section{Conclusions}

Extragenital LSA affects about $15-20 \%$ of patients with genital LSA. It is generally asymptomatic and usually affects neck, shoulders, and upper trunk, presenting as flat, white papules or slight atrophic white plaques. The diagnosis is mainly clinical and is confirmed by histopathology.

Clinically, our patient showed a specific skin lesions that made us hypothesize an inflammatory disease, such as morphea or extragenital LSA, but due to symmetrical distribution and the peculiar size, the lesions were not pathognomonic for any disease.

The uncertainty in diagnosing extragenital LSA was due to the uncommon sites of the lesions, very close to anogenital LSA, rather than on the trunk or sites of pressure. On the contrary, dermoscopy added features suggesting the hypothesis of extragenital LSA, showing well-demarcated, whitish structureless areas, and comedo-like openings [4]. Dermoscopy also allowed the differential diagnosis with morphea since the latter usually shows fibrotic beams and linear branching vessels on dermoscopy (5). A peculiar dermoscopic feature in our case was the peppered arrangement, which is usually observed only in vulvar LSA [6]: it is thought to be related to melanophages displaced in both the upper dermis and perifollicular site. Thus, peppering may represent an additional dermoscopic clue in the differential diagnosis between morphea and extragenital LSA.
Further cases of extragenital LSA need to be reported and collected, to deepen the understanding of this rare disease's dermoscopic features, confirming the presence of peppering as clue for differential diagnosis.

\section{References}

1. Lacarrubba F, Pellacani G, Verzì AE et al. Extragenital lichen sclerosus: Clinical, dermoscopic, confocal microscopy and histologic correlations. J Am Acad Dermatol. 2015;72(1) Supplement 1: 50-52. DOI:10.1016/j.jaad.2014.07.008. PMID: 25500042.

2. Kreuter A, Wischnewski J, Terras $\mathrm{S}$ et al. Coexistence of lichen sclerosus and morphea: A retrospective analysis of 472 patients with localized scleroderma from a German tertiary referral center. J Am Acad Dermatol. 2012;67(6):1157-1162. DOI: 10.1016/j. jaad.2012.04.003. PMID: 22533994.

3. Shim WH, Jwa SW, Song M et al. Diagnostic usefulness of dermatoscopy in differentiating lichen sclerous et atrophicus from morphea. J Am Acad Dermatol. 2012;66(4):690-1. DOI: 10.1016/j. jaad.2011.06.042. PMID: 22421117.

4. Garrido-Ríos AA, Álvarez-Garrido H, Sanz-Muñoz C et al. A. Dermoscopy of extragenital lichen sclerosus. Arch Dermatol. 2009;145(12):1468. DOI: 10.1001/archdermatol.2009.261. PMID:20026867.

5. Errichetti E, Stinco G. Dermoscopy in General Dermatology: A Practical Overview. Dermatol Ther. 2016;6(4):471-507. DOI: 10.1007/s13555-016-0141-6. PMID: 27613297. PMCID: PMC5120630.

6. Borghi A, Corazza M, Minghetti S et al. Dermoscopic features of vulvar lichen sclerosus in the setting of a prospective cohort of patients: New observations. Dermatol. 2016;232:71-77. DOI: 10.1159/000439198. PMID: 26574744. 